INPLASY

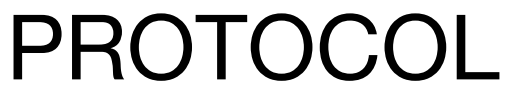

To cite: Gupta et al. Relative efficacy of biologic agents for alopecia areata: protocol for a systematic review with quantitative syntheses of the evidence base. Inplasy protocol 202220082. doi: 10.37766/inplasy2022.2.0082

Received: 19 February 2022

Published: 20 February 2022

Corresponding author: Aditya Gupta

agupta@mediproberesearch.com

Author Affiliation:

Mediprobe Research Inc.

Support: Self-financed.

Review Stage at time of this submission: Formal screening of search results.

Conflicts of interest:

None declared.

\section{Relative efficacy of biologic agents for alopecia areata: protocol for a systematic review with quantitative syntheses of the evidence base}

Gupta, AK¹; Venkataraman, M²; Bamimore, $\mathrm{MA}^{3}$.

Review question / Objective: We plan to conduct a network meta-analysis study on the relative effectiveness of biologic agents for alopecia areata.

Condition being studied: Alopecia Areata.

Eligibility criteria: Randomized and observational studies that investigated the impact of monotherapy with biologic agents for alopecia areata. Only evidence in English will be included; there will be no date restrictions.

INPLASY registration number: This protocol was registered with the International Platform of Registered Systematic Review and Meta-Analysis Protocols (INPLASY) on 20 February 2022 and was last updated on 20 February 2022 (registration number INPLASY202220082).

\section{INTRODUCTION}

Review question / Objective: We plan to conduct a network meta-analysis study on the relative effectiveness of biologic agents for alopecia areata.

Rationale: In alopecia areata (AA), immune dysfunction results in loss of hair on the scalp and/or the body. Alopecia totalis and Alopecia universalis are forms of AA; in the former, hair loss occurs throughout the entire scalp-while the loss happens throughout the entire body in the latter. Though the cause of AA is not fully elucidated, neither sex nor race has been associated with the development of $\mathrm{AA}$. While diagnosing $A A$ is easy, managing it is not: most-if not all-medicines for AA are not approved by the United States Food and Drug Administration (FDA), anddespite treatment-remission is a common occurrence. Furthermore, the existing therapies are non-curative. Biologics are 
among the recent therapeutic agents for AA. The aim of our systematic review is to determine the relative efficacy of monotherapy with biologic agents for AA.

Condition being studied: Alopecia Areata.

\section{METHODS}

Search strategy: Our systematic search will be conducted in at least two databases, namely, PubMed and Scopus.

Participant or population: Persons of any age, sex/gender and race/ethnicity-and who have a diagnosis of alopecia areata.

Intervention: Our intervention of interest is any monotherapy with a biologic agent for alopecia areata.

Comparator: Comparators can include placebo/vehicle.

Study designs to be included: Evidence will be gathered from randomized and observational studies.

Eligibility criteria: Randomized and observational studies that investigated the impact of monotherapy with biologic agents for alopecia areata. Only evidence in English will be included; there will be no date restrictions.

Information sources: Searches were conducted in at least two databases, namely, PubMed and Scopus.

Main outcome(s): The outcome(s) will be consolidated according to the evidence we gather.

Additional outcome(s): Not applicable.

Data management: Data will be organized into spreadsheets.

Quality assessment / Risk of bias analysis: Quality of evidence within studies will be assessed using Cochrane Collaboration's risk of bias (RoB) tool; evidence quality across studies will be evaluated using the Grading of Recommendations,
Assessment, Development and Evaluations (GRADE) framework.

Strategy of data synthesis: The evidence we gather will guide all quantitative analyses; if there is sufficient data, metaanalyses will be conducted.

Subgroup analysis: If there is sufficient data, clinically meaningful subgroup analyses will be done.

Sensitivity analysis: Conduct of sensitivity analyses would be dependent upon the evidence we gather.

Language: Only evidence in English language will be included.

Country(ies) involved: Canada.

Keywords: alopecia areata; biologics; meta-analysis; quality of evidence.

Contributions of each author:

Author 1 - Aditya Gupta.

Email: agupta@mediproberesearch.com Author 2 - Maanasa Venkataraman. Email: maanasa0502venkat@gmail.com Author 3 - Mary Bamimore.

Email: mbamimor@alumni.uwo.ca 